\title{
Move Based Algorithm for Runtime Mapping of Dataflow Actors on Heterogeneous MPSoCs
}

Kevin J. M. Martin Thanh Dinh Ngo Jean-Philippe Diguet

\author{
Univ. Bretagne-Sud \\ UMR CNRS 6285, Lab-STICC \\ Lorient, France
}

20/10/2017

Groupe de Travail Optimisation pour les Systèmes Intégrés

Bretagne Sud

US:

यasprice 


\section{Outline}

(1) Introduction

(2) Related work

(3) Move-based algorithm

(4) Results 


\section{Outline}

(1) Introduction

- Video Streaming

- Dataflow

- Heterogeneous Multi-processor platform

- Dataflow Mapping and scheduling

- Communication model 


\section{Introduction}

Video Streaming

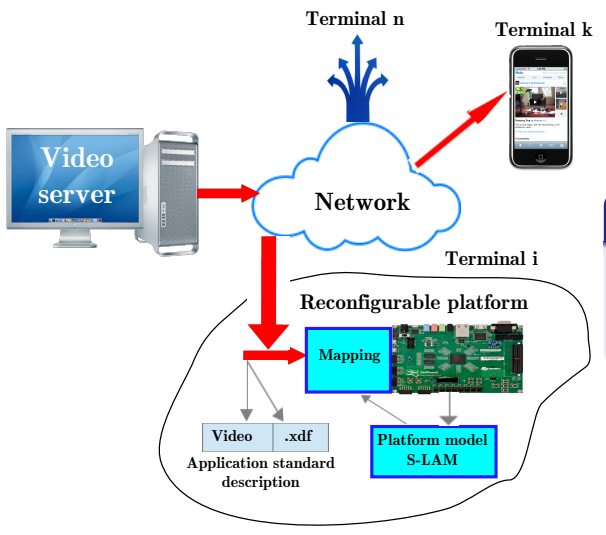

\section{Video coding}

\section{- Standards}

\section{(2) Evolution and profiles}




\section{Introduction}

\section{Dataflow}

\section{Network of actors}

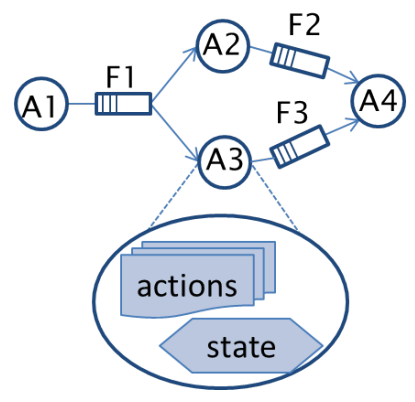

\section{Dataflow}

- Formal Model of Computation (MoC)

- Explicit spatial and temporal parallelism

- Static or dynamic actors

- Execute actions ("fire" actions)

- Firing rule

- Enough tokens in input FIFOs

- Enough space in output FIFOs 


\section{Introduction}

Dataflow Model of Computations

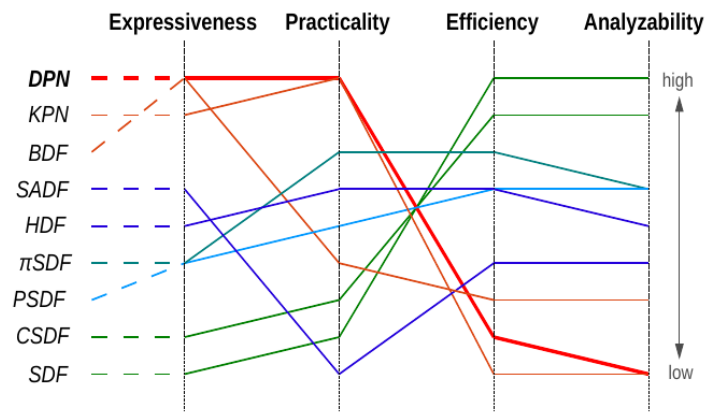

Dynamic MoC

\section{$\checkmark$ Expressivity \\ $\times$ Analyzability}

\section{Static MoCs}

$\times$ Expressivity

$\checkmark$ Analyzability 


\section{Introduction}

Heterogeneous Multi-processor platform

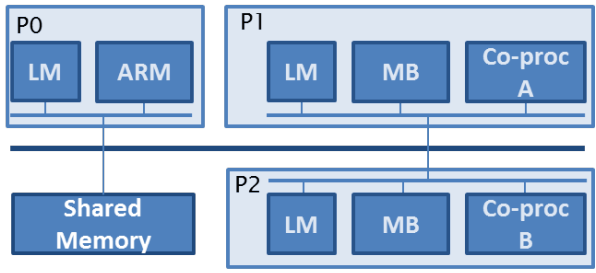

\section{Platform components}

- General purpose processors

- Custom processors (ASIP)

- Hardware accelerators

Heterogeneous MPSoC 


\section{Introduction}

Dataflow Mapping and scheduling

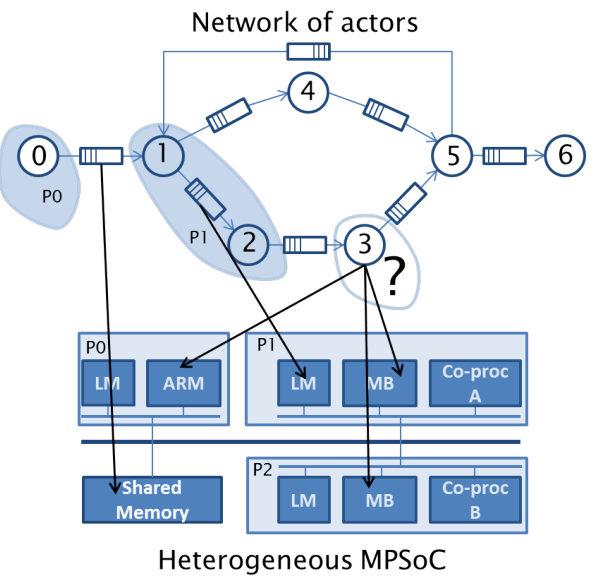

\section{Actor mapping}

- Equivalent to graph partitioning problem

- NP hard

- Computational load over processors

- Communication load/ connectivity of actors

- Platform characteristics 


\section{Introduction}

Dataflow Mapping and scheduling

\section{Different kinds of mapping}

- Static mapping (at compile time)

- On-the-fly mapping (at run-time)

- Hybrid mapping (mix of two above)

- Runtime mapping

- Runtime remapping

- Ability to change the mapping during the execution of the application 


\section{Introduction}

Communication model

\section{Analytical model}

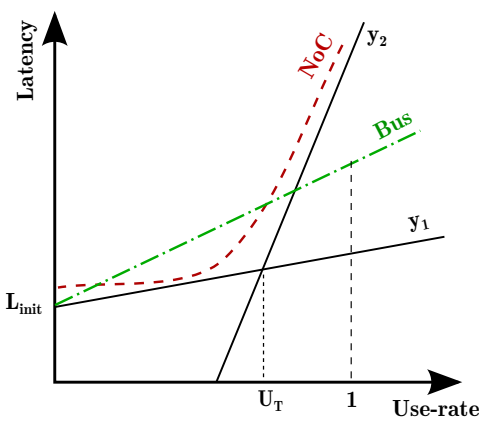

- Generic and parametric communication model

- Link between use-rate and latency

- Communications of a NoC or a bus

$f_{C l}(x)= \begin{cases}y_{1} & \text { if } x \leq \text { threshold }\left(U_{T}\right) \\ y_{2} & \text { otherwise }\end{cases}$ 


\section{Outline}

\section{(1) Introduction}

\section{(2) Related work}

(3) Move-based algorithm 


\section{Related work}

Table: Various approaches for the mapping of streaming applications

\begin{tabular}{lllll}
\hline Ref. & MoC & Platform & Comm. & Map. \\
\hline$[18],[8]-2008$ & SDF & Fixed-Homo & N/A & Static \\
{$[3]-2010$} & N/A & Generic-Heter & NoC & On-the-fly \\
{$[10]-2008$} & N/A & Fixed-Heter & Constant & On-the-fly \\
{$[17]-2013$} & SDF & Generic-Heter & NoC & Hybrid \\
{$[15]-2012$} & KPN & Fixed-Homo & NoC & Hybrid \\
{$[20]-2011$} & SADF & Generic-Heter & N/A & N/A \\
{$[21]-2013$} & DPN & Generic-Homo & Constant & Hybrid \\
{$[1]-2013$} & KPN & Generic-Heter & Yes & Hybrid \\
{$[4]-2011$} & N/A & Fixed-Homo & NoC & Hybrid \\
{$[2]-2012$} & KPN & Fixed-Heter & Yes & N/A \\
{$[16]-2010$} & N/A & Fixed-Heter & N/A & Hybrid \\
{$[7]-2012$} & SDF & Generic-Heter & IPC & Hybrid \\
{$[13]-2013$} & KPN & Generic-Homo & Constant & Hybrid \\
{$[9]-2014$} & DPN & generic-Heter & Yes & Hybrid \\
{$[14]-2015$} & KPN & Generic-Heter & Constant & Hybrid+R \\
{$[5]-2013$} & SDF & Generic-Homo & NoC & Hybrid+R \\
Ours & DPN & Generic-Heter & Yes & Hybrid+R \\
\hline
\end{tabular}




\section{Outline}

(3) Move-based algorithm

- Overview

- Parameters and evaluation metrics

- Pre-processing - PP

- Runtime mapping initialization - RMI

- Runtime remapping - RR 


\section{Move-based algorithm}

Overview

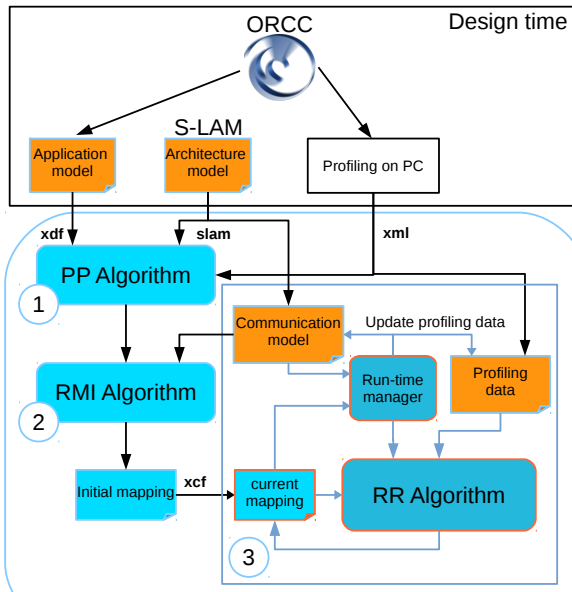

\section{At design time}

- Application model

- Architecture and communication model

- Application profiling

\section{At run time}

(1) Pre-Processing (PP)

(2) Runtime Mapping Initialisation (RMI)

(3) Runtime Remapping (RR) 


\section{Move-based algorithm}

\section{Parameters and evaluation metrics}

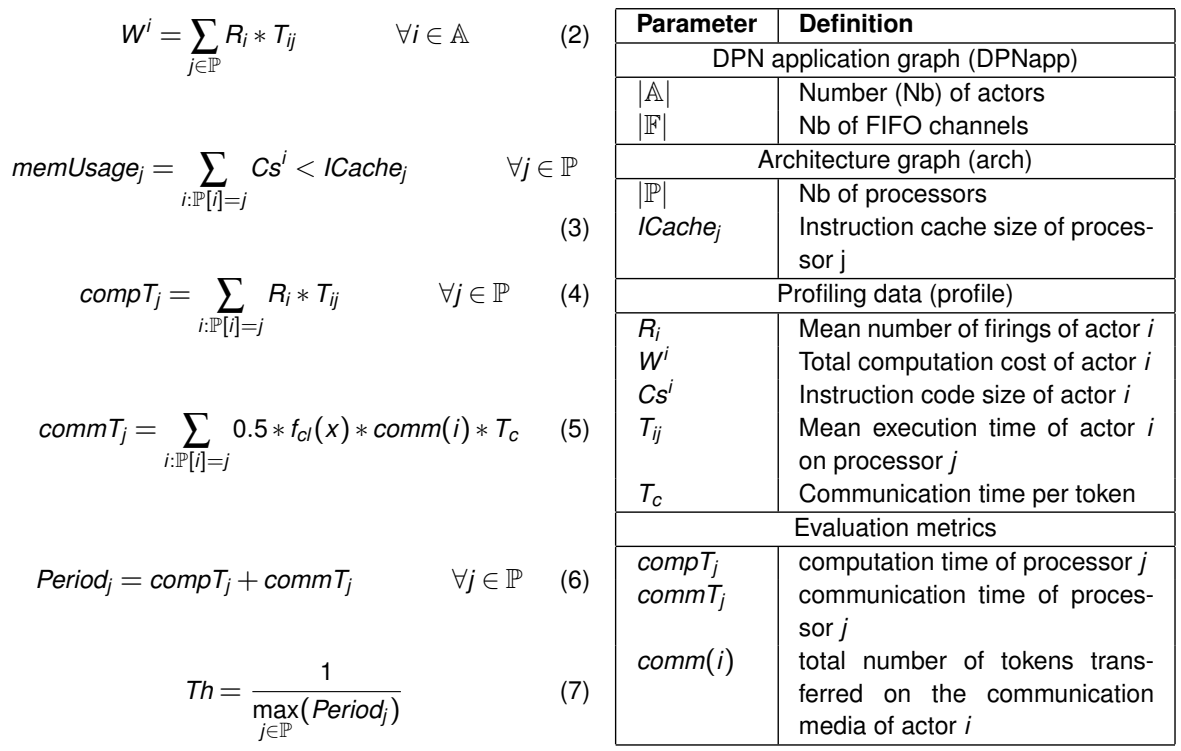




\section{Move-based algorithm}

\section{Parameters and evaluation metrics}

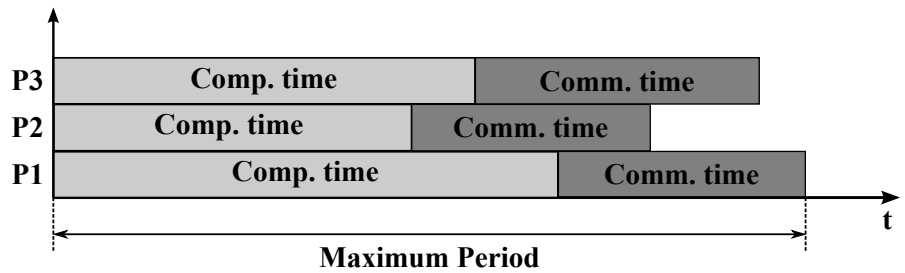

\section{Objective}

\section{Minimize the maximum period}




\section{Move-based algorithm}

Pre-processing - PP

\section{Objective}

Find the lower bound period, the unreachable lowest period to define the processing budget for all processors

\section{Assumptions}

- Profiling of the execution time of each actor on each processor 


\section{Move-based algorithm}

Runtime mapping initialization - RMI

\section{Objective}

Find rapidly a good initial mapping

\section{Greedy algorithm + speculative approach}

- relies on a factor called alpha $(\alpha)$, a ratio of processor cycle budget considered during the first step (computing-oriented) mapping

- takes into account both computation and communication workloads

- performs both actor and data mappings 


\section{Move-based algorithm}

Runtime mapping initialization - RMI

Two phases algorithm

- Computation phase

(2) Communication phase

1) Computation phase

- Sorting actors according to their total computation cost (bubble sort)

(2) Map the first actor to its best processor

(3) Update the processing use until $\alpha$

\section{2) Communication phase}

- Sorting remaining actors according to their total communication use

- actors on same processor $\Rightarrow$ communication time $=0$

- actors on different processors $\Rightarrow$ "bet" with communication model

(2) Map the first actor to the processor with least communication

(3) Update the processing use 


\section{Move-based algorithm}

Algorithm principle

\section{During application execution}

- System monitoring and application profiling (workload)

- Unbalancing load over the processors $\Rightarrow$ Remapping needed?

\section{Runtime remapping}

- Inspired by the Fiduccia and Mattheyses algorithm (FM), a famous partitioning algorithm used in VLSI [6]

- Only one actor is allowed to move from one processor to another

- Actor move $\Rightarrow$ Gain but also Cost

- Balance between migration cost and performance improvement

$$
\text { Costremap }_{\text {remax }}(\text { gainT }(i))
$$




\section{Runtime remapping - $\mathrm{RR}$}

Finding the possible moves

\section{Modified FM algorithm}

\begin{tabular}{l||l}
\multicolumn{1}{l||}{ FM algorithm } & Our algorithm \\
\hline \hline Cell move & Actor move \\
\hline 2 partitions & $n$ processors \\
\hline All cells & $\begin{array}{l}\text { Only actors on the proces- } \\
\text { sor with maximum period }\end{array}$ \\
\hline Off-line & At runtime
\end{tabular}

Consider the processor with the maximum period 


\section{Runtime remapping - $\mathrm{RR}$}

Trade-off between migration cost and performance improvement

\section{Actor move decision}

- Unlock all actors in the list of actor move candidates

(2) For each actor

- Compute the gain of performance and a migration cost

- lock actor

(3) Find the maximum gain

\section{Migration cost}

- All the binary code of actors are contained in a shared memory

- Migration cost = function of cache miss that happens when moving actor from one processor to another one 


\section{Move-based algorithm}

Runtime remapping - RR

$$
\operatorname{Cost}_{\text {mig }}(i)=f_{C l}(x) * C s^{i}
$$

$$
\operatorname{PerG}(i)=\max P e r l-\max \operatorname{PerN}(i)
$$

$$
\operatorname{gain} T(i)=N * \operatorname{PerG}(i)-\operatorname{Cost}_{m i g}(i)
$$

\section{Where}

- $C s^{i}$ : size of the binary code of actor $i$

- $f_{C l}(x)$ : communication latency

- maxPerl: maximum period before actor move

- maxPerN: maximum new period

- The cost is smoothed over $N$ periods 


\section{Outline}

(4) Results

- Setup environment

- The need of runtime remapping

- Results on MPEG4-SP 


\section{Results}

Setup environment

\section{Tools}

- SDF3 tool [19]: generated dynamic dataflow applications

- ORCC [11]: real dataflow applications

- MPEG4 Part 2 Simple Profile (MPEG4-SP)

- System-Level Architecture Model (S-LAM) [12] 


\section{Results}

The need of runtime remapping
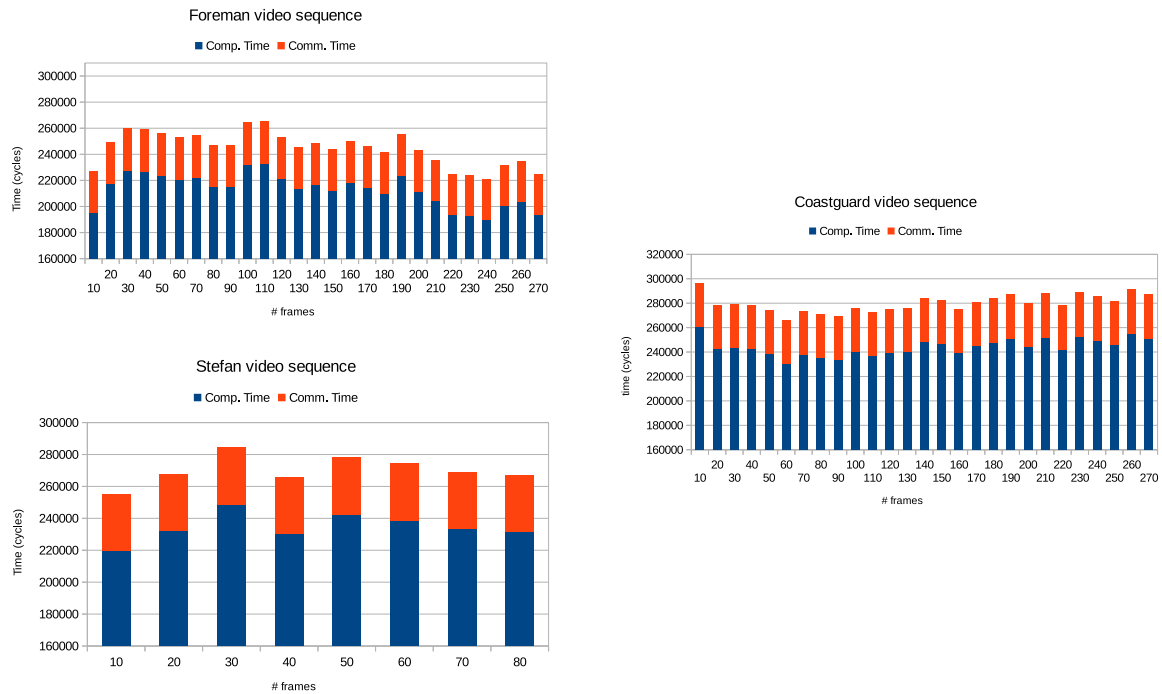


\section{Results}

Results on MPEG4-SP

\section{Properties of MPEG4-SP video decoder}

\begin{tabular}{lllll}
\hline Decoder & Profile & YUV & \#Actors & \#FIFOs \\
\hline MPEG4 Part 2 & SP & yes & 41 & 104 \\
\hline
\end{tabular}

\section{Accelerators used in platform 7.1}

\begin{tabular}{llllllll}
\hline Platform & MB1 & MB2 & MB3 & MB4 & MB5 & MB6 & MB7 \\
\hline 7.1 & Merger & IDCT & Parser & Inter & IQ+IAP & Add & IDCT
\end{tabular}

\section{Profiling workload}

- AT10: Average time updated after 10 frames

- SWT10: Sliding Window Time, last 10 frames 


\section{Results}

\section{Results on MPEG4-SP/ Foreman}

— Hybrid mapping without remapping - STW10

Move based mapping - STW10

Hybrid mapping without remapping - AT10

Move based mapping - AT10

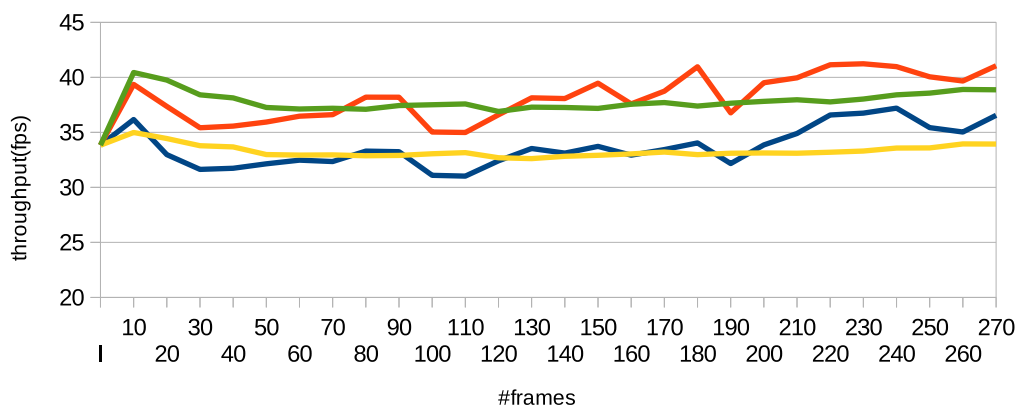




\section{Results}

\section{Results on MPEG4-SP/ Stefan}

- Hybrid mapping without remapping - STW10

Move based mapping - STW10

Hybrid mapping without remapping - AT10

Move based mapping - AT10

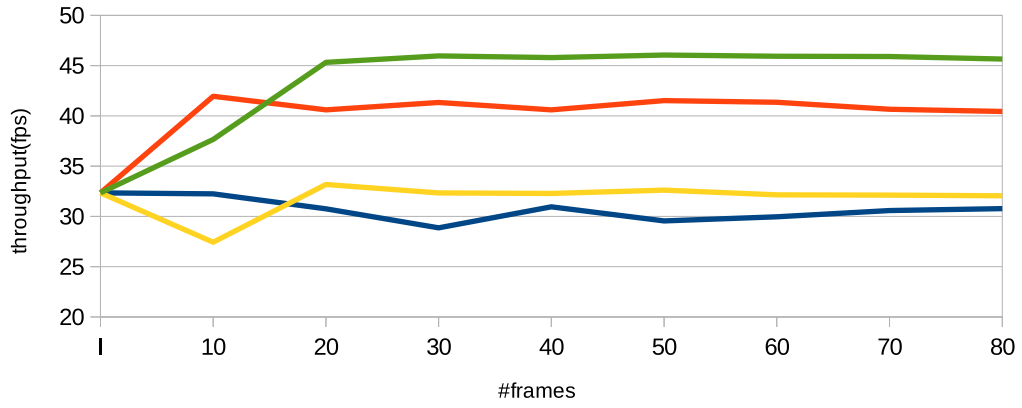




\section{Results}

Results on MPEG4-SP/ Coastguard

_ Hybrid mapping without remapping - STW10

- Move based mapping - STW10

Hybrid mapping without remapping - AT10

Move based mapping - AT10

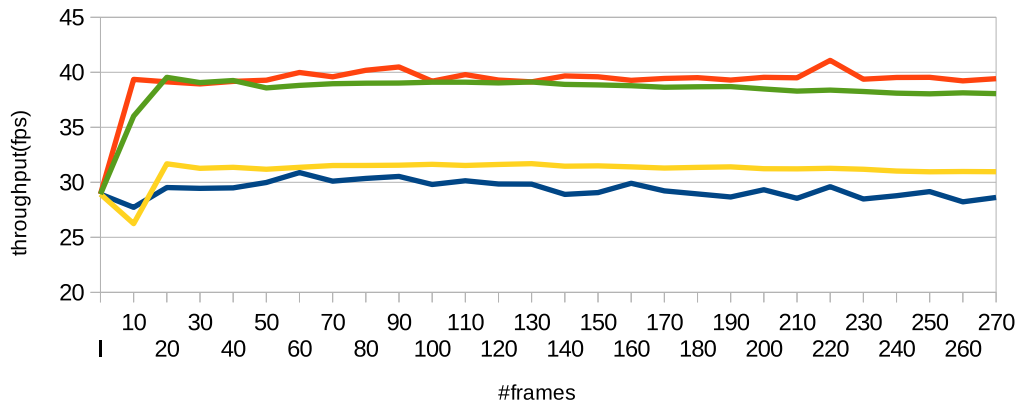




\section{Summary}

- Move based algorithm for runtime (re)mapping of dataflow actors

- Profiling / monitoring of the application

- Fast algorithm

- Take into account migration cost when moving actors 


\section{References I}

[1] J. Castrillon, R. Leupers, and G. Ascheid.

MAPS: Mapping Concurrent Dataflow Applications to Heterogeneous MPSoCs.

Industrial Informatics, IEEE Transactions on, 9(1):527-545, Feb 2013.

[2] J. Castrillon, A. Tretter, R. Leupers, and G. Ascheid.

Communication-aware mapping of KPN applications onto heterogeneous MPSoCs.

In Proceedings of the 49th Annual Design Automation Conference, DAC '12, pages 1266-1271, New York, NY, USA, 2012. ACM.

[3] E. de Souza Carvalho, N. Calazans, and F. Moraes.

Dynamic task mapping for MPSoCs.

Design Test of Computers, IEEE, 27(5):26-35, Sept 2010.

[4] S. Kaushik, A. Singh, and T. Srikanthan.

Computation and communication aware run-time mapping for NoC-based MPSoC platforms.

In SOC Conference (SOCC), 2011 IEEE International, pages 185-190, Sept 2011.

[5] C. Lee, S. Kim, and S. Ha.

Efficient run-time resource management of a manycore accelerator for stream-based applications.

In Embedded Systems for Real-time Multimedia (ESTIMedia), 2013 IEEE 11th Symposium on, pages 51-60, Oct 2013.

[6] S. K. Lim.

Practical Problems in VLSI Physical Design Automation.

Springer Publishing Company, Incorporated, 1 edition, 2008.

[7] J. Lin, A. Gerstlauer, and B. L. Evans.

Communication-aware heterogeneous multiprocessor mapping for real-time streaming systems.

Journal of Signal Processing Systems, 69(3):279-291, Dec. 2012.

[8] W. Liu, M. Yuan, X. He, Z. Gu, and X. Liu.

Efficient sat-based mapping and scheduling of homogeneous synchronous dataflow graphs for throughput optimization.

In Real-Time Systems Symposium, 2008.

[9] D.-T. Ngo, J.-P. Diguet, K. Martin, and D. Sepulveda.

Communication-model based Embedded Mapping of Dataflow Actors on Heterogeneous MPSoC.

In Proceedings of the Conference on Design and Architectures for Signal and Image Processing (DASIP), 2014. 


\section{References II}

[10] V. Nollet, P. Avasare, H. Eeckhaut, D. Verkest, and H. Corporaal.

Run-Time Management of a MPSoC Containing FPGA Fabric Tiles.

Very Large Scale Integration (VLSI) Systems, IEEE Transactions on, 16(1):24-33, Jan 2008.

[11] ORCC.

The Open RVC-CAL Compiler : A Development Framework for Dataflow Programs.

[12] M. Pelcat, J. F. Nezan, J. Piat, J. Croizer, and S. Aridhi.

A System-Level Architecture Model for Rapid Prototyping of Heterogeneous Multicore Embedded Systems.

In Conference on Design and Architectures for Signal and Image Processing (DASIP) 2009, page 8 pages, nice, France, Sept. 2009.

[13] W. Quan and A. D. Pimentel.

A scenario-based run-time task mapping algorithm for MPSoCs.

In Proceedings of the 50th Annual Design Automation Conference, DAC '13, pages 131:1-131:6, New York, NY, USA, 2013. ACM.

[14] W. Quan and A. D. Pimentel.

A Hybrid Task Mapping Algorithm for Heterogeneous MPSoCs.

ACM Trans. Embed. Comput. Syst., 14(1):14:1-14:25, Jan. 2015.

[15] L. Schor, I. Bacivarov, D. Rai, H. Yang, S.-H. Kang, and L. Thiele.

Scenario-based design flow for mapping streaming applications onto on-chip many-core systems.

In Proceedings of the 2012 International Conference on Compilers, Architectures and Synthesis for Embedded Systems, CASES '12, New York, NY, USA, 2012. ACM.

[16] A. Schranzhofer, J.-J. Chen, L. Santinelli, and L. Thiele.

Dynamic and adaptive allocation of applications on MPSoC platforms.

In Design Automation Conference (ASP-DAC), 201015 th Asia and South Pacific, pages 885-890, Jan 2010.

[17] A. K. Singh, A. Kumar, and T. Srikanthan.

Accelerating throughput-aware runtime mapping for heterogeneous MPSoCs.

ACM Trans. Des. Autom. Electron. Syst., 18(1), 2013.

[18] S. Stuijk, M. Geilen, and T. Basten.

Exploring trade-offs in buffer requirements and throughput constraints for synchronous dataflow graphs.

In 43rd ACM/IEEE DAC, pages 899-904, 2006. 


\section{References III}

[19] S. Stuijk, M. Geilen, and T. Basten.

Sdf3: Sdf for free.

In 6th Int. Conf. on Application of Concurrency to System Design,, 2006.

[20] S. Stuijk, M. Geilen, B. Theelen, and T. Basten.

Scenario-aware dataflow: Modeling, analysis and implementation of dynamic applications.

In Int. Conf on Embedded Computer Systems (SAMOS), 2011.

[21] H. Yviquel, E. Casseau, M. Raulet, P. Jääskeläinen, and J. Takala.

Towards run-time actor mapping of dynamic dataflow programs onto multi-core platforms.

In Int. Symp. on Image and Signal Processing and Analysis (ISPA), France, 2013. 